\title{
ON THE EDIT DISTANCE FUNCTION OF THE RANDOM GRAPH
}

\author{
RYAN R. MARTIN AND ALEXANDER W.N. RIASANOVSKY
}

\begin{abstract}
Given a hereditary property of graphs $\mathcal{H}$ and a $p \in$ $[0,1]$, the edit distance function $\operatorname{ed}_{\mathcal{H}}(p)$ is asymptotically the maximum proportion of edge-additions plus edge-deletions applied to a graph of edge density $p$ sufficient to ensure that the resulting graph satisfies $\mathcal{H}$. The edit distance function is directly related to other well-studied quantities such as the speed function for $\mathcal{H}$ and the $\mathcal{H}$-chromatic number of a random graph.

Let $\mathcal{H}$ be the property of forbidding an Erdős-Rényi random graph $F \sim \mathbb{G}\left(n_{0}, p_{0}\right)$, and let $\varphi$ represent the golden ratio. In this paper, we show that if $p_{0} \in[1-1 / \varphi, 1 / \varphi]$, then a.a.s. as $n_{0} \rightarrow \infty$,

$$
\operatorname{ed}_{\mathcal{H}}(p)=(1+o(1)) \frac{2 \log n_{0}}{n_{0}} \cdot \min \left\{\frac{p}{-\log \left(1-p_{0}\right)}, \frac{1-p}{-\log p_{0}}\right\} .
$$
\end{abstract}

Moreover, this holds for $p \in[1 / 3,2 / 3]$ for any $p_{0} \in(0,1)$.

\section{INTRODUCTION}

All graphs are finite and simple, i.e., without loops and multi-edges. A graph is nonempty if it has at least one edge. Denote $P_{n}$ to be the path graph on $n$ vertices.

For any $p \in[0,1]$ and any positive integer $n$, write $\mathbb{G}(n, p)$ to be the distribution of graphs according to the Erdős-Rényi random graph model with edge probability $p$. That is, $G \sim \mathbb{G}(n, p)$ means that the event that $u v \in E(G)$ for $u v \in\left(\begin{array}{c}\{1, \ldots, n\} \\ 2\end{array}\right)$ are independent and identically distributed (i.i.d.) with common probability $p$. We write a.a.s. to mean that a sequence of events holds with probability approaching 1 under some implied limit. The limit will be clear by the context. All

2010 Mathematics Subject Classification. 05C35,05C80.

Key words and phrases. edit distance, speed function, colored regularity graphs, random graph.

Both authors' research was partially supported by NSF award DMS-1839918 (RTG). Martin's was partially supported by Simons Foundation Collaboration Grant \#353292. 
logarithms are natural unless explicitly stated otherwise.

1.1. Edit distance results and forbidding a random graph. The edit distance measures the minimum number of "edits" (that is, edgeadditions plus edge-deletions) sufficient to turn one graph into another. This metric has been studied in contexts such as property testing and evolutionary biology (see [3,11]). Formally, for any two $n$-vertex graphs $G, H$ on the same vertex set,

$$
\operatorname{dist}(G, H)=|E(G) \triangle E(H)| \cdot\left(\begin{array}{c}
n \\
2
\end{array}\right)^{-1}
$$

where $\triangle$ is the symmetric difference operation for sets.

A graph property $\mathcal{H}$ is hereditary if $\mathcal{H}$ is closed under isomorphism and vertex deletion. For any family $\mathcal{F}$ of graphs, we may write $\operatorname{Forb}(\mathcal{F})$ for the hereditary property of graphs which do not contain an induced copy of $F$ for any $F \in \mathcal{F}$. Any hereditary property is of the form $\operatorname{Forb}(\mathcal{F})$ for some $\mathcal{F}$. A hereditary property of the form $\operatorname{Forb}(\{F\})$ for a single graph $F$ is called a principal hereditary property and we will write $\operatorname{Forb}(F)$ for simplicity.

A hereditary property $\mathcal{H}$ is nontrivial if, for every positive integer $n$, there exists a graph in $\mathcal{H}$ of order $n$. All hereditary properties in this paper are nontrivial. If $\mathcal{H}$ is a nontrivial hereditary property, then for all graphs $G$, we define

$$
\operatorname{dist}(G, \mathcal{H})=\min \{\operatorname{dist}(G, H): \exists H \in \mathcal{H} \text { s.t. } V(H)=V(G)\} .
$$

An early result that has motivated subsequent research is as follows:

Theorem 1 (Alon-Stav [2]). For a nontrivial hereditary property $\mathcal{H}$, there exists a $p^{*}=p_{\mathcal{H}}^{*} \in[0,1]$ so that with $G \sim \mathbb{G}\left(n, p^{*}\right)$,

$$
\lim _{n \rightarrow \infty} \max _{|V(G)|=n} \operatorname{dist}(G, \mathcal{H})=\mathbb{E}[\operatorname{dist}(G, \mathcal{H})]+o(1) .
$$

In other words, random graphs of density $p^{*}$ asymptotically achieve the maximum distance to $\mathcal{H}$. For any $p \in[0,1]$ and any property $\mathcal{H}$ nontrivial and hereditary, let

$$
\operatorname{ed}_{\mathcal{H}}(p):=\limsup _{n \rightarrow \infty} \max _{\substack{|V(G)|=n, e(G)=\left\lfloor p\left(\begin{array}{l}
n \\
2
\end{array}\right)\right\rfloor}} \operatorname{dist}(G, \mathcal{H}) .
$$


We call $\operatorname{ed}_{\mathcal{H}}$ the edit distance function of $\mathcal{H}$. Theorem 2 below demonstrates that the maximum distance $\mathcal{H}$ among all density- $p$ graphs is achieved asymptotically by Erdős-Rényi random graphs of expected density $p$.

Theorem 2 (Balogh-Martin [4]). Let $\mathcal{H}$ be a nontrivial hereditary property. For all $p \in[0,1]$, if $G \sim \mathbb{G}(n, p)$, then

$$
\operatorname{ed}_{\mathcal{H}}(p)=\lim _{n \rightarrow \infty} \mathbb{E}[\operatorname{dist}(G, \mathcal{H})]
$$

Moreover the function $\operatorname{ed}_{\mathcal{H}}$ is continuous and concave-down.

Proposition 3 below has several short proofs and follows from Bollobás' asymptotic result on the chromatic number of a random graph (see [5]), together with established techniques for computing edit distance functions (see [11]).

Proposition 3 (Alon-Stav 2]). Let $F \sim \mathbb{G}\left(n_{0}, 1 / 2\right)$ and define $\mathcal{H}:=$ $\operatorname{Forb}(F)$. Then a.a.s. with $n_{0} \rightarrow \infty$,

$$
\operatorname{ed}_{\mathcal{H}}(p)=(1+o(1)) \frac{2 \log _{2} n_{0}}{n_{0}} \cdot \min \{p, 1-p\}
$$

Our main result extends Proposition 3 so that we are able to determine the edit distance function asymptotically for all $p_{0}$ in a relatively large open interval around $1 / 2$. Let $\varphi=(1+\sqrt{5}) / 2$ be the golden ratio. Note that $1-\varphi^{-1} \approx 0.381966$ and $\varphi^{-1} \approx 0.618034$.

Theorem 4. Fix $p_{0} \in(0,1)$, let $F \sim \mathbb{G}\left(n_{0}, p_{0}\right)$, and define $\mathcal{H}:=$ $\operatorname{Forb}(F)$. If $p_{0} \in\left[1-\varphi^{-1}, \varphi^{-1}\right]$ then a.a.s. with $n_{0} \rightarrow \infty$,

$$
\operatorname{ed}_{\mathcal{H}}(p)=(1+o(1)) \frac{2 \log n_{0}}{n_{0}} \cdot \min \left\{\frac{p}{-\log \left(1-p_{0}\right)}, \frac{1-p}{-\log p_{0}}\right\}
$$

holds for all $p \in[0,1]$. If $p_{0} \in\left[0,1-\varphi^{-1}\right)$, then a.a.s. (2) holds for all $p \in[1 / 3,1]$. If $p_{0} \in\left(\varphi^{-1}, 1\right]$, then a.a.s. (2) holds for all $p \in[0,2 / 3]$.

In fact, the $o(1)$ error term depends only on the constant $p_{0}$ and holds uniformly for all $p$ in each of the respective intervals. 
The first author conjectured (see [11]) that for all $p_{0} \in[0,1]$, (2) holds a.a.s. for all $p \in[0,1]$. Theorem 4 proves this for a range of $p_{0}$ of size $\approx 0.236068$.

1.2. Equivalent parameters. The edit distance function is also interesting because of its connection to other parameters involving random graphs. For $\mathcal{H}$ any nontrivial hereditary property and any $p \in(0,1)$, the speed of $\mathcal{H}$ is

$$
c_{\mathcal{H}}(p):=\lim _{k \rightarrow \infty}-\log _{2}(\mathbb{P}[\mathbb{G}(k, p) \in \mathcal{H}]) \cdot\left(\begin{array}{l}
n \\
2
\end{array}\right)^{-1}
$$

Indeed, this limit does exist and a proof of that fact appears in [1] and in [6]. See also the survey [11].

The following observation was made by Thomason but it can be shown to follow from a prior result due to Bollobás and Thomason [6].

Theorem 5 (Thomason [13]). Let $\mathcal{H}$ be a nontrivial hereditary property. Then for all $p \in(0,1)$,

$$
c_{\mathcal{H}}(p)=\left(-\log _{2}(p(1-p))\right) \cdot \operatorname{ed}_{\mathcal{H}}\left(\frac{\log (1-p)}{\log (p(1-p))}\right) .
$$

Note that the function $f:(0,1) \rightarrow(0,1)$ defined by

$$
f(x):=\frac{\log (1-x)}{\log (x(1-x))}
$$

on $x \in(0,1)$ is invertible. Since $\operatorname{ed}_{\mathcal{H}}$ is continuous, $c_{\mathcal{H}}$ can be computed from $\operatorname{ed}_{\mathcal{H}}$ and vice versa. As a result, combining Theorem 5 with Theorem 4 yields a result on the speed function of hereditary properties defined by random graphs.

Corollary 6. Fix $p_{0} \in(0,1)$, let $F \sim \mathbb{G}\left(n_{0}, p_{0}\right)$, and define $\mathcal{H}:=$ $\operatorname{Forb}(F)$. If $p_{0} \in\left[1-\varphi^{-1}, \varphi^{-1}\right]$ then a.a.s. with $n_{0} \rightarrow \infty$,

$$
c_{\mathcal{H}}(p)=(1+o(1)) \frac{2 \log _{2} n_{0}}{n_{0}} \cdot \min \left\{\frac{\log (1-p)}{\log \left(1-p_{0}\right)}, \frac{\log p}{\log p_{0}}\right\}
$$

holds for all $p \in[0,1]$. If $p_{0} \in\left[0,1-\varphi^{-1}\right)$, then a.a.s. (3) holds for all $p \in\left[1-\varphi^{-1}, 1\right]$. If $p_{0} \in\left(\varphi^{-1}, 1\right]$, then a.a.s. (3) holds for all $p \in\left[0, \varphi^{-1}\right]$. 
For any hereditary property $\mathcal{H}$ and any graph $G$, let $\chi_{\mathcal{H}}(G)$ be the $\mathcal{H}$-chromatic number of $G$. This is the minimum nonnegative integer $k$ for which there exists a partition $V(G)=V_{1} \cup \cdots \cup V_{k}$ such that $G\left[V_{i}\right]$ satisfies $\mathcal{H}$ for all $i \in\{1, \ldots, k\}$. If $\mathcal{H}$ is the property of being an empty graph, then $\chi_{\mathcal{H}}(G)$ is the chromatic number of $G$.

Bollobás and Thomason established Theorem 7 for the $\mathcal{H}$-chromatic number of a random graph.

Theorem 7 (Bollobás-Thomason [6]). Let $p \in(0,1)$ and let $\mathcal{H}$ be a nontrivial hereditary property. Then a.a.s. with $G \sim \mathbb{G}(n, p)$,

$$
\chi_{\mathcal{H}}(G)=(1+o(1)) c_{\mathcal{H}}(p) \frac{n}{2 \log _{2} n}
$$

Bollobás' classic asymptotic result [5] on the chromatic number of the random graph can be derived from Theorem 7 by observing that if $\mathcal{H}_{\mathrm{em}}$ is the property of being an empty graph, then $c_{\mathcal{H}}(p)=-\log _{2}(1-p)$ and so $\chi_{\mathcal{H}_{\mathrm{em}}}(G)=(1+o(1)) \frac{n}{2 \log _{1 /(1-p)} n}$ a.a.s.

However, the fact that $c_{\mathcal{H}}(p)=-\log _{2}(1-p)$ can itself be derived from Theorem 5 and the entirely trivial observation that $\operatorname{ed}_{\mathcal{H}_{\mathrm{em}}}(p)=p$. In general, $\chi_{\mathcal{H}}$ has a close relationship with both $c_{\mathcal{H}}$ and $\operatorname{ed}_{\mathcal{H}}$.

The rest of the paper is organized as follows: In Section 2, we discuss colored regularity graphs (CRGs) and prove some basic results that have the potential to apply to a wide variety of edit distance results beyond the scope of this paper. In Section 3, we give the proof of Theorem 4. Section 4 includes a proof of the fact that for all $p \in\left[1-\varphi^{-1}, \varphi^{-1}\right], \operatorname{ed}_{\mathcal{H}}(p)$ can be computed by a set CRGs whose order is bounded by a constant depending only on $\mathcal{H}$. Section 4 also includes a discussion of the role paths play in CRGs. In Section 5, we discuss open questions and potential future work.

\section{Colored Regularity GraphS}

In this section, we address colored regularity graphs. In Section 2.1, we address background and basic facts about colored regularity graphs. Section 2.2 discusses the new notion of $p$-prohibited CRGs. Lemma 20 
and Lemma 23 are important new results on $p$-prohibited CRGs. They are proven in Section 2.3 and Section 2.4, respectively.

2.1. Background on CRGs. The key element to studying the edit distance problem is the colored regularity graph, which was defined by Alon and Stav [2] but appeared as types in the prior literature by Bollobás and Thomason (see [6]).

Definition 8. A colored regularity graph $K$ is a complete graph, together with a partition $V(K)=\mathrm{VW}(K) \cup \mathrm{VB}(K)$ of the vertex set into white and black vertices, and a partition $E(K)=\mathrm{EW}(K) \cup \mathrm{EB}(K) \cup$ $\mathrm{EG}(K)$ of the edge set into white, black, and gray edges.

A $C R G K^{\prime}$ is called a sub-CRG of $C R G K$ (denoted $K^{\prime} \subseteq K$ ) if $K^{\prime}$ is obtained by deleting some vertices from $K$ and all incident edges.

CRGs approximate large graphs and we want to know whether a forbidden graph $F$ is in a graph approximated by a given CRG. We express this in terms of embeddings of graphs into CRGs.

Definition 9. A graph $F$ embeds into $C R G K$ (written $F \mapsto K$ ) if there exists a function $\phi: V(F) \rightarrow V(K)$ such that:

- If $u v \in E(F)$, then either $\phi(u)=\phi(v) \in \mathrm{VB}(K)$, or $\phi(u) \phi(v) \in$ $\mathrm{EB}(K) \cup \mathrm{EG}(K)$

- If $u v \in E\left(F^{c}\right)$, then either $\phi(u)=\phi(v) \in \mathrm{VW}(K)$, or $\phi(u) \phi(v) \in$ $\mathrm{EW}(K) \cup \mathrm{EG}(K)$.

For any CRG $K$, we will treat the elements of $\mathbb{R}^{V(K)}$ both as functions on $V(K)$ and as vectors indexed by the vertices of $K$. For any two such $\mathbf{x}, \mathbf{y} \in \mathbb{R}^{V(K)}$, we define $\langle\mathbf{x}, \mathbf{y}\rangle:=\sum_{u \in V(K)} \mathbf{x}(u) \mathbf{y}(u)$. We also let $M_{K}(p) \in \mathbb{R}^{V(K) \times V(K)}$ be the matrix whose $u v$-th entry is

$m_{u v}:=\left\{\begin{aligned} p, & u \neq v \text { and } u v \in \operatorname{EW}(K), \text { or } u=v \text { and } u \in \operatorname{VW}(K) \\ 1-p, & u \neq v \text { and } u v \in \operatorname{EB}(K), \text { or } u=v \text { and } u \in \operatorname{VB}(K) \\ 0, & u \neq v \text { and } u v \in \operatorname{EG}(K) .\end{aligned}\right.$

The all-ones vector $\mathbf{1} \in \mathbb{R}^{V(K)}$ is defined by $\mathbf{1}(u)=1$ for all $u \in V(K)$ and the all-zeroes vector is just $\mathbf{0}=0 \cdot \mathbf{1}$. Furthermore, we let $\Delta_{K}$ be the standard simplex associated to $K$ which consists of all $\mathbf{x} \in \mathbb{R}^{V(K)}$ so that $\mathbf{x} \geq \mathbf{0}$ in the component-wise sense and $\langle\mathbf{x}, \mathbf{1}\rangle=1$. The elements 
of $\Delta_{K}$ will be called weight vectors.

Now define

$$
\begin{aligned}
g_{K}(p, \mathbf{x}) & :=\left\langle\mathbf{x}, M_{K}(p) \mathbf{x}\right\rangle \text { and } \\
g_{K}(p) & :=\min \left\{g_{K}(p, \mathbf{x}): \mathbf{x} \in \Delta_{K}\right\} .
\end{aligned}
$$

A weight vector $\mathbf{x} \in \Delta_{K}$ is said to be optimal for $K$ if $g_{K}(p, \mathbf{x})=g_{K}(p)$. For any $p \in[0,1]$, a CRG $K$ is said to be $p$-core if for any optimal weight vector $\mathbf{x}, \mathbf{x}(u)>0$ for all $u \in V(K)$. It follows that for $K$ a $p$-core $\mathrm{CRG}$, there exists a unique optimal weight vector.

For any hereditary property $\mathcal{H}=\operatorname{Forb}(\mathcal{F})$ we define the following family of CRGs:

$$
\mathcal{K}_{\mathcal{H}}:=\{K \text { a CRG }: F \not \neg K \text { for all } F \in \mathcal{F}\} .
$$

Theorem 10 is the main technique for computing $\operatorname{ed}_{\mathcal{H}}(p)$, hence understanding the set $\mathcal{K}_{\mathcal{H}}$ is crucial to understanding $\operatorname{ed}_{\mathcal{H}}(p)$. The first equality was given by Balogh and Martin [4] and the second by Marchant and Thomason [10].

Theorem 10. Let $\mathcal{H}$ be a nontrivial hereditary property. Then for all $p \in[0,1]$,

$$
\operatorname{ed}_{\mathcal{H}}(p)=\inf _{K \in \mathcal{K}_{\mathcal{H}}} g_{K}(p)=\min _{K \in \mathcal{K}_{\mathcal{H}}} g_{K}(p)
$$

It follows by definition that the minimum in Theorem 10 is obtained by a $p$-core CRG and as Theorem 11 shows, $p$-core CRGs have a welldefined structure.

Theorem 11 (Marchant-Thomason [10]). Let $p \in[0,1]$ and suppose $K$ is a $p$-core $C R G$.

(a) If $p \in[0,1 / 2]$, then $\mathrm{EB}(K)=\emptyset$ and for all $u v \in \mathrm{EW}(K), u, v \in$ $\operatorname{VB}(K)$.

(b) If $p \in[1 / 2,1]$, then $\mathrm{EW}(K)=\emptyset$ and for all $u v \in \mathrm{EB}(K), u, v \in$ $\mathrm{VW}(K)$.

To summarize, if $p \leq 1 / 2$, then a $p$-core CRG has no black edges and all white edges must be between black vertices. If $p \geq 1 / 2$, then 
a $p$-core CRG has no white edges and all black edges must be between white vertices. As a result, if $p=1 / 2, p$-core CRGs have neither black nor white edges.

Remark 12. A CRG $K$ is 1/2-core if and only if all edges of $K$ are gray.

2.2. $p$-prohibited CRGs. In this paper, we introduce the notion of a prohibited CRG.

Definition 13. For any $p \in[0,1]$ and any $C R G J$, we say that $J$ is p-prohibited if for any $p$-core $C R G K, J$ is not a sub-CRG of $K$.

For example, Theorem 11 shows that if $p \in[0,1 / 2)$, then the only 2 -vertex CRGs that are not $p$-prohibited are those with a gray edge or the CRG with two black vertices and a white edge. See Figure 1 .

Remark 14. There is an abundance of CRGs which are neither p-core nor p-prohibited. For example, consider the CRGs $K$ and $K^{\prime}$ defined as follows. Let $K$ consist of 3 black vertices with 2 white edges and 1 gray edge. For all $p \in[0,1], K$ is not $p$-core. Now let $K^{\prime}$ be the $C R G$ on 4 black vertices whose white edges induce a $P_{4}$ and all other edges are gray. Clearly $K^{\prime}$ contains $K$. It is an exercise to see that $K^{\prime}$ is $p$-core for all $p \in\left[0,1-\varphi^{-1}\right)$, so $K$ is not $p$-prohibited on this interval.
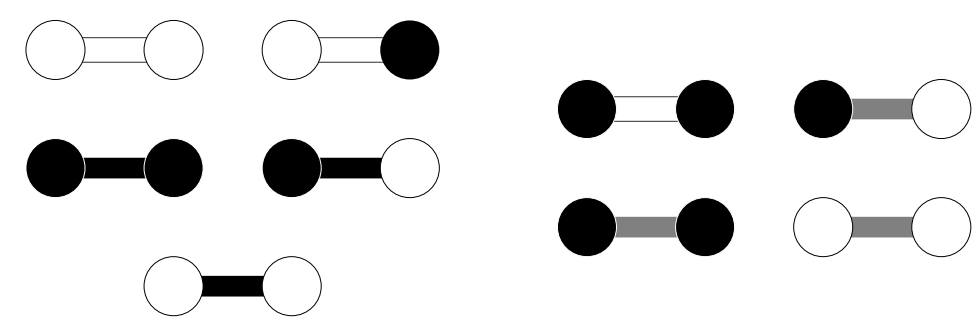

Figure 1. All two-vertex CRGs. The five on the left are $p$-prohibited for all $p \in[0,1 / 2]$. The four on the right are $p$-core for all $p \in[0,1 / 2)$. 
We also want to introduce the notion of the complement of a CRG.

Definition 15. If $K$ is a $C R G$, then the complement of $K$ is the unique $C R G \bar{K}$, such that

- $\mathrm{VW}(\bar{K})=\mathrm{VB}(K), \mathrm{VB}(\bar{K})=\mathrm{VW}(K)$,

- $\mathrm{EW}(\bar{K})=\operatorname{EB}(K), \operatorname{EB}(\bar{K})=\operatorname{EW}(K)$, and $\operatorname{EG}(\bar{K})=\operatorname{EG}(K)$.

For a graph $G$, the notation is $G^{c}$ is used to denote the graph complement, so as to avoid confusion. There is symmetry in the edit distance function about $p=1 / 2$ with respect to complements.

Proposition 16. If $p \in[0,1]$ and $K$ is a $C R G$, then $g_{K}(p)=g_{\bar{K}}(1-p)$.

Proof. This follows from the equality of the matrices $M_{K}(p)=M_{\bar{K}}(1-$ $p)$ :

$$
\begin{aligned}
g_{K}(p) & =\min \left\{\left\langle\mathbf{x}, M_{K}(p) \mathbf{x}\right\rangle: \mathbf{x} \in \Delta_{K}\right\} \\
& =\min \left\{\left\langle\mathbf{x}, M_{\bar{K}}(1-p) \mathbf{x}\right\rangle: \mathbf{x} \in \Delta_{K}\right\}=g_{\bar{K}}(1-p) .
\end{aligned}
$$

There is also symmetry in the edit distance function about $p=1 / 2$ when it comes to $p$-prohibition.

Proposition 17. For all $p \in[0,1]$, a CRG $J$ is p-prohibited if and only if $\bar{J}$ is $(1-p)$-prohibited.

Proof. Suppose $J$ is $p$-prohibited but $\bar{J}$ is not $(1-p)$-prohibited. Then, there is a $(1-p)$-core CRG $\bar{K}$ that contains $\bar{J}$ as a sub-CRG. If $K$ is not $p$-core, then there is a $K^{\prime} \subseteq K$ such that $g_{K^{\prime}}(p)=g_{K}(p)$, but Proposition 16 gives that $g_{\overline{K^{\prime}}}(1-p)=g_{\bar{K}}(1-p)$, a contradiction to $\bar{K}$ being $(1-p)$-core.

Next, we introduce terminology which is useful in describing the structure of $p$-core and $p$-prohibited CRGs.

Definition 18. Let $K$ be a $C R G$. 
- The underlying graph of $K$ is the graph $G=(V(K), \operatorname{EB}(K) \cup$ $\mathrm{EW}(K))$.

- A component of $K$ is a component of the underlying graph of $K$.

- A disjoint union of vertex-disjoint CRGs $J, K$, denoted $J \oplus$ $K$, is a $C R G$ with vertex set $V_{J} \oplus V_{K}$, where the sub-CRG induced on $V_{J}$ is isomorphic to $J$, the sub-CRG induced on $V_{K}$ is isomorphic to $K$, and every edge incident to a vertex in each of $V_{J}$ and in $V_{K}$ has color gray. The disjoint union of $k$ copies of $K$ is $k \cdot K$.

- Let $G$ be a nonempty graph. The $C R G, K$, associated to $G$ is defined as follows: If $p \in[0,1 / 2]$, then $\mathrm{VW}(K)=\mathrm{EB}(K)=\emptyset$, $\mathrm{VB}(K)=V(G), \operatorname{EW}(K)=E(G)$, and $\operatorname{EG}(K)=E(\bar{G})$. If $p \in(1 / 2,1]$, then $\operatorname{VB}(K)=\operatorname{EW}(K)=\emptyset, \operatorname{VW}(K)=V(G)$, $\mathrm{EB}(K)=E(G)$, and $\mathrm{EG}(K)=E(\bar{G})$.

We associate CRGs to graphs for the purposes of discussing $p$-core CRGs. See Figure 2 for an example. Since 1/2-core CRGs are precisely those which have only gray edges, the definition of the CRG associated to a graph for $p=1 / 2$ is made purely out of convenience.

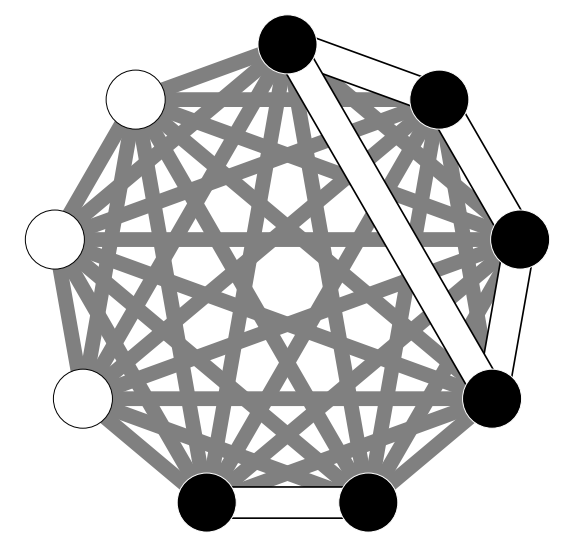

Figure 2. A CRG with 5 components. One component is the CRG associated to the cycle $C_{4}$ for $p \in[0,1 / 2)$. The edges satisfy the necessary conditions from Theorem 11 for a $p$-core CRG with $p \in[0,1 / 2)$.

In order to apply Lemma 20 below, we need the minimum adjacency eigenvalue to be at most -1 . This occurs for all nonempty graphs. 
See [7] for a more detailed discussion about eigenvalues associated to graphs.

Proposition 19. Every nonempty graph that is not disjoint cliques has minimum adjacency eigenvalue at most $-\sqrt{2}$. If a nonempty graph consists of disjoint cliques, its minimum adjacency eigenvalue is -1 . An empty graph has all adjacency eigenvalues zero.

Lemma 20. Let $G$ be a nonempty graph and let $\lambda \leq-1$ be the minimum eigenvalue of the adjacency matrix of $G$. The $C R G$ associated to $G$ is p-prohibited for all

$$
p \in\left[\frac{1}{1-\lambda}, 1-\frac{1}{1-\lambda}\right] .
$$

In Section 2.3, we prove Lemma 20. First, we need some essential terms.

Definition 21. For any positive integer $t$, the t-dalmatian $\boldsymbol{C R G}$ is the $C R G$, denoted $D_{t}$, consisting of $t$ black vertices and all edges white. The $\infty$-dalmatian $\boldsymbol{C R G}$ is the $C R G$, denoted $D_{\infty}$, which is a single white vertex. The set of $C R G$ s denoted by $\mathcal{D}_{p}$ is as follows:

- If $p \in[0,1 / 2)$, then $\mathcal{D}_{p}$ is the set of all CRGs whose components are dalmatian CRGs.

- If $p \in(1 / 2,1]$, then $\mathcal{D}_{p}$ is the set of all CRGs whose components are complements of dalmatian CRGs.

- If $p=1 / 2$, then $\mathcal{D}_{1 / 2}$ is the set of all CRGs whose components are single vertices.

See Figure 3 for dalmatian CRGs of small order.
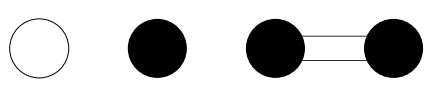

Figure 3. The dalmatian CRGs $D_{\infty}=\overline{D_{1}}, D_{1}=$ $\overline{D_{\infty}}, D_{2}, D_{3}$, and $D_{4}$.
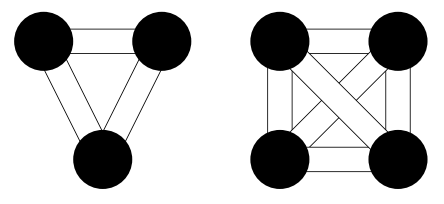
Remark 22. For all $p \in[0,1]$ and each $K \in \mathcal{D}_{p}, K$ is a p-core $C R G$. Moreover, if $p \in[0,1 / 2]$, then $g_{D_{\infty}}(p)=p$ and for each positive integer $t$,

$$
g_{D_{t}}(p)=\min _{\mathbf{x} \in \Delta_{D_{t}}}\left\langle\mathbf{x}, M_{D_{t}}(p) \mathbf{x}\right\rangle=\frac{1}{t^{2}}\left\langle\mathbf{1}, M_{D_{t}}(p) \mathbf{1}\right\rangle=p+\frac{1-2 p}{t} .
$$

If $p \in[1 / 2,1]$, then $g_{\overline{D_{\infty}}}(p)=1-p$ and for each positive integer $t$, $g_{\overline{D_{t}}}(p)=1-p+\frac{2 p-1}{t}$.

In Lemma 23, we show that for all $p \in\left[1-\varphi^{-1}, \varphi^{-1}\right]$, the only $p$-core CRGs are those that belong to $\mathcal{D}_{p}$.

Lemma 23. For $p \in[0,1]$, the $C R G$ associated to $P_{3}$ is p-prohibited if and only if $p \in\left[1-\varphi^{-1}, \varphi^{-1}\right]$. In particular for all $p \in\left[1-\varphi^{-1}, \varphi^{-1}\right]$, a CRG $K$ is $p$-core if and only if $K \in \mathcal{D}_{p}$.

Remark 24. Since the minimum eigenvalue of the adjacency matrix of $P_{3}$ is $-\sqrt{2}$, Lemma 20 gives that $P_{3}$ is prohibited for $p$ in the interval $[\sqrt{2}-1,2-\sqrt{2}] \approx[0.414,0.586]$. Lemma [23, however, gives that $P_{3}$ is prohibited on the larger interval $\left[1-\varphi^{-1}, \varphi^{-1}\right] \approx[0.382,0.618]$.

In Section 2.4, we prove Lemma 23.

2.3. Proof of Lemma 20. The result from Theorem 25] below was originally shown by Sidorenko [12 using different language and has appeared in several other forms throughout the study of hereditary properties. See [11] for a more detailed history. For convenience, we state it in the language of CRGs.

Theorem 25. Let $K$ be a p-core $C R G$ with optimal weight vector $\mathbf{x} \in$ $\Delta_{K}$. Then

$$
M_{K}(p) \mathbf{x}=g \mathbf{1} .
$$


So, Theorem 25 establishes that the optimal weight vector produces a weighting that is balanced.

Lemma 26. Let $0 \leq p \leq 1$ and $J$ be a CRG. If there exists a nonzero vector $\boldsymbol{\delta} \in \mathbb{R}^{V(J)}$ so that $\langle\boldsymbol{\delta}, \mathbf{1}\rangle=0$ and

$$
\left\langle\boldsymbol{\delta}, M_{J}(p) \boldsymbol{\delta}\right\rangle \leq 0
$$

then $J$ is p-prohibited.

Proof. We proceed by contradiction. Suppose $J$ is not $p$-prohibited and that there exists $\boldsymbol{\delta}$ as above. Then there exists a $p$-core CRG $K$ containing $J$ and we may let $\mathbf{x}$ denote the optimal weight vector for $K$. We extend $\boldsymbol{\delta}$ to a vector $\boldsymbol{\delta}^{\prime} \in \mathbb{R}^{V(K)}$ by letting $\boldsymbol{\delta}^{\prime}(u):=0$ if $u \in V(K) \backslash V(J)$ and $\boldsymbol{\delta}^{\prime}(u):=\boldsymbol{\delta}(u)$ if $u \in V(J)$. Note that $\left\langle\boldsymbol{\delta}^{\prime}, \mathbf{1}\right\rangle=0$.

Since $\mathbf{x}$ is the optimal weight vector for the $p$-core CRG $K, \mathbf{x}(u)>0$ for all $u \in V(K)$ and it follows that there exists some $\varepsilon>0$ so that $\mathbf{x}^{\prime}:=\mathbf{x}+\varepsilon \boldsymbol{\delta}^{\prime}$ lies in $\Delta_{K}$ and $\mathbf{x}^{\prime}(u)=0$ for some $u \in V(K)$. By the definition of $g_{K}(p)$, the fact that $\mathbf{x}$ is optimal, and Theorem 25 ,

$$
\begin{aligned}
0 & <g_{K}\left(p, \mathbf{x}^{\prime}\right)-g_{K}(p, \mathbf{x}) \\
& =\left\langle\mathbf{x}+\varepsilon \boldsymbol{\delta}^{\prime}, M_{K}(p)\left(\mathbf{x}+\varepsilon \boldsymbol{\delta}^{\prime}\right)\right\rangle-\left\langle\mathbf{x}, M_{K}(p) \mathbf{x}\right\rangle \\
& =2 \varepsilon\left\langle\boldsymbol{\delta}, M_{K}(p) \mathbf{x}\right\rangle+\varepsilon^{2}\left\langle\boldsymbol{\delta}^{\prime}, M_{K}(p) \boldsymbol{\delta}^{\prime}\right\rangle \\
& =2 \varepsilon\left\langle\boldsymbol{\delta}^{\prime}, g_{K}(p) \mathbf{1}\right\rangle+\varepsilon^{2}\left\langle\boldsymbol{\delta}, M_{J}(p) \boldsymbol{\delta}\right\rangle \\
& =\varepsilon^{2}\left\langle\boldsymbol{\delta}, M_{J}(p) \boldsymbol{\delta}\right\rangle \leq 0,
\end{aligned}
$$

a contradiction to the assumption that $J$ is not $p$-prohibited.

We include one more well-known fact about $p$-core CRGs.

Proposition 27 (See [11]). Let $K_{1}, \ldots, K_{\ell}$ be CRGs and let $K=$ $K_{1} \oplus \cdots \oplus K_{\ell}$. Then for all $p \in[0,1]$,

$$
g_{K}(p)^{-1}=\sum_{i=1}^{\ell} g_{K_{i}}(p)^{-1} .
$$

In particular, $K$ is $p$-core if and only if each of $K_{1}, \ldots, K_{\ell}$ are $p$-core.

Lemma 28. Let $p \in[0,1]$. A CRG $J$ is p-prohibited if and only if for all p-core $C R G s K$ and all positive integers $k$, the $C R G(k \cdot J) \oplus K$ is p-prohibited. 
Proof. To prove the forward implication, if $J$ is $p$-prohibited, then no $p$-core CRG can contain $k \cdot J$ because it would contain $J$. To prove the reverse implication, if $J$ is not $p$-prohibited then there exists a $p$-core CRG, $L$, containing $J$. Then $J \oplus K$ is contained in $L \oplus K$, which is $p$-core by Proposition 27, as desired.

With the primary tools of Lemmas 26 and 28 established, we now proceed to prove Lemma 20 itself.

Recall that $J$ is the CRG associated to a nonempty graph $G$. If $p=1 / 2$, then by Theorem [11, $J$ is $1 / 2$-prohibited if and only if $J$ has an edge that is not gray. Thus, any nonempty $G$ gives that $J$ is $1 / 2$-prohibited, settling the case where $p=1 / 2$.

Now suppose $p \in(0,1 / 2)$. Write $A$ for the adjacency matrix of $G$ and suppose $A \mathbf{x}=\lambda \mathbf{x}$ for some unit vector $\mathbf{x}$ where $\lambda$ is the minimum eigenvalue of $A$. Then $J$ is the the CRG on $V(G)$ with all vertices black, and where edge $u v$ is white if $u v \in E(G)$, and $u v$ is gray if $u v \in E\left(G^{c}\right)$. So $M_{J}(p)=(1-p) I+p A$.

For convenience, we write $V_{1}$ and $V_{2}$ for the vertex sets in $2 \cdot J$ corresponding to each copy of $J$. Let $\boldsymbol{\delta} \in \mathbb{R}^{V(2 \cdot J)}$ be defined by

$$
\boldsymbol{\delta}(u):=\left\{\begin{aligned}
\mathbf{x}(u), & u \in V_{1} \\
-\mathbf{x}(u), & u \in V_{2}
\end{aligned}\right.
$$

By definition, $\langle\mathbf{1}, \boldsymbol{\delta}\rangle=0$. Moreover note that

$$
\begin{aligned}
\left\langle\boldsymbol{\delta}, M_{2 \cdot J}(p) \boldsymbol{\delta}\right\rangle & =\left\langle\mathbf{x}, M_{J}(p) \mathbf{x}\right\rangle+\left\langle-\mathbf{x}, M_{J}(p)(-\mathbf{x})\right\rangle \\
& =2\left\langle\mathbf{x}, M_{J}(p) \mathbf{x}\right\rangle \\
& =2\langle\mathbf{x},((1-p) I+p A) \mathbf{x}\rangle \\
& =2(1-p)\langle\mathbf{x}, I \mathbf{x}\rangle+2 p\langle\mathbf{x}, A \mathbf{x}\rangle \\
& =2(1-p)\langle\mathbf{x}, \mathbf{x}\rangle+2 p \lambda\langle\mathbf{x}, \mathbf{x}\rangle \\
& =2 \cdot(1-(1-\lambda) p) \cdot\langle\mathbf{x}, \mathbf{x}\rangle
\end{aligned}
$$

If $p \geq 1 /(1-\lambda)$, then $\left\langle\boldsymbol{\delta}, M_{2 \cdot J}(p) \boldsymbol{\delta}\right\rangle \leq 0$. By Lemma 26, the CRG $2 \cdot J$ is $p$-prohibited and by Lemma $28, J$ itself is $p$-prohibited. This 
settles the case where $p \in(0,1 / 2)$.

Finally, for the case of $p \in(1 / 2,1)$, Proposition 17 gives that $J$ is $p$-prohibited if and only if $J$ is $(1-p)$-prohibited. This concludes the proof of Lemma 20.

2.4. Proof of Lemma 23. Lemma 29 below is a result in pure graph theory that is reminiscent of the theorem that categorizes $\left\{P_{4}, C_{4}, C_{4}^{c}\right\}$ free graphs as threshold graphs. A dominant vertex in a graph is one for which every other vertex is its neighbor.

Lemma 29. If $G$ is a connected $\left\{P_{4}, C_{4}\right\}$-free graph, then $G$ has a dominant vertex.

Proof. Let $u$ be a vertex of $G$ which attains the maximum degree $\Delta=\Delta(G)$ and let $A:=N_{G}(u)$ and $B:=V(G) \backslash(A \cup\{u\})$. If $B=\emptyset$, then $u$ is the desired vertex, so we assume otherwise. Let $w \in B$. Since $G$ avoids induced $P_{4^{-}}$s, connectivity implies $G$ has diameter at most 2 .

In particular, $\operatorname{dist}_{G}(u, w)=2$, so there exists some vertex $v \in A$ so that $u v w$ is an induced path on 3 vertices. If $v^{\prime}$ is any vertex in $A \backslash\{v\}$, then $v^{\prime} u v w$ is a path on 4 vertices. Since $u w \notin E(G)$ and $G$ avoids both induced $P_{4}$-s and induced $C_{4}$-s, it follows that $v v^{\prime} \in E(G)$. So $v$ is adjacent to $\{u, w\} \cup(A \backslash\{v\})$ and has degree at least $\Delta+1$, a contradiction.

Lemma 29 yields a very strong structural theorem on CRGs, Lemma 30. Recall the definition of the underlying graph of CRG, $K$, in Definition 18, the graph whose vertices are the vertices of $K$ and whose edges are the non-gray edges of $K$.

Lemma 30. Let $p \in[1 / 3,2 / 3]$. If a $p$-core $C R G$ has an underlying graph which is $\left\{P_{4}, C_{4}\right\}$-free, then every component of the underlying graph is a clique. That is, every component of such a CRG must be a member of $\mathcal{D}_{p}$.

Proof. If $p=1 / 2$, then as we saw in Remark 12, a $p$-core CRG has only gray edges and so the underlying graph is empty.

Let $p \in[1 / 3,1 / 2)$. Every trivial component of the underlying graph is simply a vertex in the CRG. Let $K$ be a nontrivial component of 
the CRG. By Theorem [11, the vertices of $K$ must be black and by Lemma 29, the underlying graph of $K$ has a dominant vertex $u$.

Let $\mathbf{x} \in \Delta_{K}$ be the optimal weight vector for $K$ and define $g:=$ $g_{K}(p)$. By Theorem [25, $M_{K}(p) \mathbf{x}=g \mathbf{1}$ and by inspecting the entry indexed by $u$,

$$
g=(1-p) \mathbf{x}(u)+p \cdot \sum_{u \neq v \in V(K)} \mathbf{x}(v)=p+(1-2 p) \mathbf{x}(u)>p .
$$

For a contradiction, we now suppose $K$ is not a dalmatian CRG. Hence, there exists some gray edge $v w$, and the sub-CRG $K^{\prime}$ on $v$ and $w$ is the disjoint union of two black vertices. Since $K$ is $p$-core,

$$
g<g_{K^{\prime}}(p)=\min _{\mathbf{y} \in \Delta_{K^{\prime}}}(1-p)\left(\mathbf{y}(v)^{2}+\mathbf{y}(w)^{2}\right)=\frac{1-p}{2} .
$$

Altogether, $p<g<(1-p) / 2$ which implies that $p<1 / 3$, a contradiction.

The case where $p \in(1 / 2,2 / 3]$ follows by symmetry.

We now prove Lemma 23itself with the primary tool being Lemma 30. As mentioned in Remark 14, we leave it as an exercise to verify that the CRG associated with $P_{4}$ is $p$-core for $p \in\left(0,1-\varphi^{-1}\right) \cup\left(\varphi^{-1}, 1\right)$. Hence, $P_{3}$ is not $p$-prohibited in this range, proving the forward implication.

For the reverse implication, let $p \in\left[1-\varphi^{-1}, \varphi^{-1}\right]$. Since the minimum eigenvalues of the adjacency matrices of $C_{4}$ and $P_{4}$ are -2 and $-\varphi^{-1}$ respectively, Lemma 20 implies that the CRGs associated with $C_{4}$ and $P_{4}$ are $p$-prohibited for $p \in\left[1-\varphi^{-1}, \varphi^{-1}\right]$.

Suppose $K$ is a $p$-core CRG. Since $p \in[1 / 3,2 / 3]$, it follows from Lemma 30 that the components of the underlying graph of $K$ are cliques. No such graph contains an induced $P_{3}$ and so $P_{3}$ is $p$-prohibited for all $p \in\left[1-\varphi^{-1}, \varphi^{-1}\right]$, as desired.

For the second statement of the theorem, since $P_{3}$ is $p$-prohibited, the only underlying graphs of a $p$-core CRG can be disjoint cliques, which is exactly the condition of being in $\mathcal{D}_{p}$. As observed in Remark 22, all CRGs in $\mathcal{D}_{p}$ are $p$-core. This concludes the proof of Lemma 23. 


\section{Proof of The MAIN RESUlT}

To proceed with the proof of Theorem 4, we need some preparation. In Section 3.1, Lemma 31 shows that for all $p \in(1 / 3,2 / 3)$ and for any CRG $K$, there exists a sub-CRG $K^{\prime}$ of $K$ so that $g_{K^{\prime}}(p)$ is close to $g_{K}(p)$ and $K^{\prime}$ has components whose order is bounded by a function of $p$ and a tolerance term $\varepsilon$.

For the remaining discussion, let $p_{0} \in(0,1)$ and define

$$
p^{*}:=\frac{\log \left(1-p_{0}\right)}{\log \left(p_{0}\left(1-p_{0}\right)\right)} .
$$

In Section 3.2, we investigate when a random graph $F \sim \mathbb{G}\left(n_{0}, p_{0}\right)$ embeds into a CRG (i.e., when $F \mapsto K$ ). There we show that a.a.s., if a CRG, $K$, has bounded components in the above sense and the random graph does not map into $K$, then $g_{K}\left(p^{*}\right)$ has to be at least the desired value to within a small tolerance. Applying Lemma [31, this is true even if the components of $K$ are not bounded.

Finally in Section 3.3, we put together these ideas to prove our main result.

3.1. Trimming $p$-core CRGs. The main result in this subsection is Lemma 31, which establishes that, for $p \in(1 / 3,2 / 3)$, a CRG has a sub-CRG with bounded component sizes and a negligible change in the value of the $g$-function.

Lemma 31. Fix $p \in(1 / 3,2 / 3)$ and $\varepsilon \in(0,1)$. There exists a positive integer $B=B(p, \varepsilon)$ such that the following holds: For all CRGs $K$, there exists a p-core sub-CRG $K^{\prime}$ whose components have order at most $B$, and $g_{K^{\prime}}(p) \leq(1+\varepsilon) g_{K}(p)$.

The first part of the proof is to remove vertices from a CRG $K$ oneby-one in a way which does not affect $g_{K}(p)$ too much. Once enough vertices are removed, we show that each remaining vertex is incident to a bounded number of non-gray edges. Finally, we use Lemma 20 to bound the diameter of $p$-core CRGs on the interval $p \in(1 / 3,2 / 3)$. The underlying graph has bounded degree and diameter, thus its connected components have bounded order.

The proof consists of a sequence of propositions:

Proposition 32. Fix $p \in[0,1]$ and suppose $K$ is a p-core $C R G$ with least two vertices. If $\mathbf{x} \in \Delta_{K}$ is the optimal weight vector for $K$, i.e., 


$$
\begin{aligned}
& g=g_{K}(p)=g_{K}(p, \mathbf{x}) \text {, then for all } u \in V(K), \\
& \qquad g_{K \backslash\{u\}}(p) \leq g+\frac{\mathbf{x}(u)^{2}}{(1-\mathbf{x}(u))^{2}} .
\end{aligned}
$$

Proof. Let $K^{\prime}:=K \backslash\{u\}$. Since $K$ is $p$-core with at least two vertices, $\mathbf{x}(u)<1$ and we may define $\mathbf{x}^{\prime} \in \Delta_{K}$ by

$$
\mathbf{x}^{\prime}(v):=\left\{\begin{array}{cl}
0, & v=u \\
\frac{\mathbf{x}(v)}{1-\mathbf{x}(u)}, & \text { otherwise }
\end{array} .\right.
$$

In other words, if $\mathbf{e}_{u} \in \mathbb{R}^{V(K)}$ is the indicator vector for the vertex $u$, then $(1-\mathbf{x}(u)) \mathbf{x}^{\prime}=\mathbf{x}-\mathbf{x}(u) \mathbf{e}_{u}$. Recall that $M_{K}(p)$ denotes the weighted adjacency matrix of $K$. By Theorem $25, M_{K}(p) \mathbf{x}=g \mathbf{1}$ and SO

$$
\begin{aligned}
(1-\mathbf{x}(u))^{2}\left\langle\mathbf{x}^{\prime}, M_{K}(p) \mathbf{x}^{\prime}\right\rangle & =\left\langle\mathbf{x}-\mathbf{x}(u) \mathbf{e}_{u}, M_{K}(p)\left(\mathbf{x}-\mathbf{x}(u) \mathbf{e}_{u}\right)\right\rangle \\
& =\langle\mathbf{x}, g \mathbf{1}\rangle-2 \mathbf{x}(u)\left\langle\mathbf{e}_{u}, g \mathbf{1}\right\rangle+\mathbf{x}(u)^{2}\left\langle\mathbf{e}_{u}, M_{K}(p) \mathbf{e}_{u}\right\rangle \\
& \leq g-2 g \mathbf{x}(u)+\mathbf{x}(u)^{2} .
\end{aligned}
$$

By definition of $g_{K^{\prime}}(p)$ and since $\mathbf{x}^{\prime}(u)=0$,

$$
\begin{aligned}
g_{K^{\prime}}(p) & \leq g_{K}\left(p, \mathbf{x}^{\prime}\right) \\
& =\frac{\left\langle\mathbf{x}^{\prime}, M_{K}(p) \mathbf{x}^{\prime}\right\rangle}{(1-\mathbf{x}(u))^{2}} \\
& =\frac{g-2 g \mathbf{x}(u)+\mathbf{x}(u)^{2}}{(1-\mathbf{x}(u))^{2}} \\
& =g+\frac{(1-g) \mathbf{x}(u)^{2}}{(1-\mathbf{x}(u))^{2}} \\
& \leq g+\frac{\mathbf{x}(u)^{2}}{(1-\mathbf{x}(u))^{2}},
\end{aligned}
$$

which completes the proof.

Proposition 33. Fix $p \in[0,1]$ and $\varepsilon \in(0,1)$. If $K$ is a $p$-core $C R G$ with $g=g_{K}(p)$, then there exists a p-core sub-CRG $K^{\prime}$ of $K$ such that the following holds:

(1) $\left|V\left(K^{\prime}\right)\right| \leq 4 /(\varepsilon g)$,

(2) $g_{K^{\prime}}(p) \leq(1+17 \varepsilon) g$, and

(3) if $\mathbf{x}^{\prime} \in \Delta_{K^{\prime}}$ is the optimal weight vector for $K^{\prime}$, then

$$
\min _{u \in V\left(K^{\prime}\right)} \mathbf{x}^{\prime}(u) \geq \varepsilon g \text {. }
$$


Proof. Define a finite sequence of sub-CRGs

$$
K=K_{0} \supset K_{1} \supset \cdots \supset K_{\ell}
$$

as follows: First let $K:=K_{0}$ and $g_{0}:=g_{K}(p)$. For any $k \geq 0$ so that $\left|V\left(K_{k}\right)\right| \geq 2$, do the following:

(i) Let $\mathbf{x}_{k} \in \Delta_{K_{k}}$ be the optimal weight vector for $K_{k}$, i.e., $g_{K_{k}}\left(p, \mathbf{x}_{k}\right)=$ $g_{K_{k}}(p)$.

(ii) Let $u_{k} \in V\left(K_{k}\right)$ so that $\mathbf{x}_{k}\left(u_{k}\right)=\min \left\{\mathbf{x}_{k}(v): v \in V\left(K_{k}\right)\right\}$.

(iii) Let $K_{k+1}$ be any $p$-core sub-CRG of $K_{k} \backslash\left\{u_{k}\right\}$.

Since each step removes at least one vertex, $\ell \leq|V(K)|$. For each $k \in\{0, \ldots, \ell\}$, denote $g_{k}:=g_{K_{k}}(p)$.

Let $a \in\{0, \ldots, \ell\}$ be the minimum index $a$ so that $\left|V\left(K_{a}\right)\right| \leq 4 /(\varepsilon g)$. By definition of $a$ and by the fact that $\varepsilon, g<1$,

$$
\mathbf{x}_{k}\left(u_{k}\right) \leq 1 /\left|V\left(K_{k}\right)\right| \leq(\varepsilon g) / 4 \leq 1 / 4
$$

for all $k \in\{0, \ldots, a-1\}$. By Proposition 32,

$$
g_{k+1} \leq g_{k}+\frac{\mathbf{x}_{k}\left(u_{k}\right)^{2}}{\left(1-\mathbf{x}_{k}\left(u_{k}\right)\right)^{2}} \leq g_{k}+\frac{\mathbf{x}_{k}\left(u_{k}\right)^{2}}{(3 / 4)^{2}}<g_{k}+\frac{2}{\left|V\left(K_{k}\right)\right|^{2}} .
$$

Because $\left|V\left(K_{k}\right)\right| \geq\left|V\left(K_{a-1}\right)\right|+(a-1-k)$ for all $k \in\{0, \ldots, a-1\}$,

$$
\begin{aligned}
g_{a} & <g+\sum_{k=0}^{a-1} \frac{2}{\left|V\left(K_{k}\right)\right|^{2}} \\
& \leq g+\sum_{k=0}^{a-1} \frac{2}{\left(\left|V\left(K_{a-1}\right)\right|+(a-1-k)\right)^{2}} \\
& <g+\sum_{i=\left|V\left(K_{a-1}\right)\right|}^{\infty} \frac{2}{i^{2}} \\
& <g+\int_{i=\left|V\left(K_{a-1}\right)\right|-1}^{\infty} \frac{2}{x^{2}} d x \\
& =g+\frac{2}{\left|V\left(K_{a-1}\right)\right|-1} \\
& \leq g+\frac{2}{\lfloor 4 /(\varepsilon g)\rfloor} .
\end{aligned}
$$

Since $\varepsilon g<1$, it is the case that $\lfloor 4 /(\varepsilon g)\rfloor>2 /(\varepsilon g)$. Consequently,

$$
g_{a} \leq(1+\varepsilon) g \text {. }
$$


Let $b$ be the least index $a \leq b \leq \ell$ so that $\mathbf{x}_{b}(u) \geq \varepsilon g$ for all $u \in$ $V\left(K_{b}\right)$. Note that $b$ is well-defined since $\mathbf{x}_{\ell}(u)=1>\varepsilon g$ where $x$ is the optimal weighting for $K_{\ell}$, a CRG with a single vertex. For any $k \in\{a, \ldots, b-1\}$, it is the case that $\mathbf{x}_{k}\left(u_{k}\right)<\varepsilon g$ and that $\mathbf{x}_{k}\left(u_{k}\right) \leq$ $1 /\left|V\left(K_{k}\right)\right| \leq 1 / 2$ and again by Proposition 32 ,

$$
g_{k+1} \leq g_{k}+\frac{\mathbf{x}_{k}\left(u_{k}\right)^{2}}{\left(1-\mathbf{x}_{k}\left(u_{k}\right)\right)^{2}}<g_{k}+\frac{(\varepsilon g)^{2}}{(1 / 2)^{2}}=g_{k}+4 \varepsilon^{2} g^{2} .
$$

Finally by (10) and since $b-a \leq\left|V\left(K_{a}\right)\right| \leq 4 /(\varepsilon g)$,

$$
g_{b} \leq g_{a}+\sum_{k=a}^{b-1} 4 \varepsilon^{2} g^{2} \leq(1+\varepsilon) g+\left|V\left(K_{a}\right)\right| \cdot 4 \varepsilon^{2} g^{2} \leq(1+17 \varepsilon) g .
$$

Letting $K^{\prime}:=K_{b}$, we have the desired sub-CRG.

Proposition 34 below implies that we can decrease the degree of the underlying graph of a CRG $K$ without changing $g_{K}(p)$ too much.

Proposition 34. Fix $p \in(0,1)$ and $\varepsilon \in(0,1)$. If $K$ is a p-core $C R G$, then there exists a p-core sub-CRG $K^{\prime}$ of $K$ so that

(1) $g_{K^{\prime}}(p) \leq(1+\varepsilon) g_{K}(p)$, and

(2) for each $u \in V\left(K^{\prime}\right)$, $u$ is incident in $K^{\prime}$ to at most

$$
17 \varepsilon^{-1} \cdot \max \left\{\frac{1}{p}, \frac{1}{1-p}\right\}
$$

black or white edges.

Proof. We prove the claim for all $p \in(0,1 / 2]$. By duality (that is, by replacing $K$ with $\bar{K}$ and $p$ with $1-p$ ) the claim holds also for all $p \in[1 / 2,1)$. By Proposition 33 applied to $K$ and $\varepsilon / 17$, there exists a sub-CRG $K^{\prime}$ of $K$ so that $g_{K^{\prime}}(p) \leq(1+\varepsilon) g_{K}$ and whose optimal weight vector $\mathbf{x} \in \Delta_{K^{\prime}}$ has $\mathbf{x}(u) \geq \varepsilon g / 17$ for all $u \in V\left(K^{\prime}\right)$. By Theorem [11, the white vertices of $K^{\prime}$ are incident to no white or black edges. So it suffices to prove the desired inequality for black vertices. Suppose $u \in \operatorname{VB}\left(K^{\prime}\right)$. By Theorem 25, $M_{K^{\prime}}(p) \mathbf{x}=g \mathbf{1}$ and it follows that

$$
\frac{g}{p}>\frac{g}{p}-\frac{1-p}{p} \mathbf{x}(u)=\sum_{u v \in \operatorname{EW}\left(K^{\prime}\right)} \mathbf{x}(v) \geq \frac{\varepsilon g}{17} \cdot\left|\left\{v: u v \in \operatorname{EW}\left(K^{\prime}\right)\right\}\right| .
$$

So the number of vertices adjacent to a vertex via a non-gray edge is at most $17 /(\varepsilon p)$, as desired. 
Next, we uniformly bound the diameter of all $p$-core CRGs, for each $p \in(1 / 3,2 / 3)$.

Proposition 35. For all positive integers d, the CRG associated to the path $P_{d}$ on $d$ vertices is p-prohibited for all

$$
p \in\left[\frac{1}{1+2 \cos (\pi /(d+1))}, 1-\frac{1}{1+2 \cos (\pi /(d+1))}\right] .
$$

Proof. It is a well-known fact from spectral graph theory that the spectrum of the adjacency matrix of $P_{d}$, the path on $d$ vertices is the multiset $\left\{2 \cos \left(\frac{\pi k}{d+1}\right): k \in\{1, \ldots, d\}\right\}$. See, for example, [8]. In particular, the minimum such eigenvalue is $-2 \cos (\pi /(d+1))$. Lemma 20 gives that $P_{d}$ is $p$-prohibited for $p$ in the stated range.

Finally, we prove Lemma 31 .

Proof of Lemma 31. Since the sequence $\left\{\frac{1}{1+2 \cos (\pi /(d+1))}\right\}$ is monotone decreasing and converges to $1 / 3$, there exists a positive integer $d=d_{p}$ so that $P_{d}$ is $p$-prohibited. Let $K^{\prime}$ be the sub-CRG from Proposition 34 and write $G$ for its underlying graph. Then by construction, $G$ has degree at most

$$
D=17 \varepsilon^{-1} \cdot \max \left\{\frac{1}{p}, \frac{1}{1-p}\right\} .
$$

Let $C$ be any component of $G$. Since $P_{d}$ is $p$-prohibited, $C$ has diameter at most $d-1$. It follows that

$$
|C| \leq 1+D+D(D-1)+\cdots+D(D-1)^{d-1}=: B(p, \varepsilon),
$$

as desired.

3.2. Forbidding a random graph. Now we proceed to prove our main result, Theorem 4. First, recall that Theorem 7 says that if $F^{\prime} \sim \mathbb{G}\left(n_{0}^{\prime}, p_{0}\right)$ then a.a.s.,

$$
\chi_{H}\left(F^{\prime}\right)=(1+o(1)) c_{\mathcal{H}}\left(p_{0}\right) \frac{n_{0}^{\prime}}{2 \log _{2} n_{0}^{\prime}} .
$$

This is useful because in the proof of Lemma [37, we repeatedly decompose induced subgraphs of a random graph of order $n_{0} \gg n_{0}^{\prime}$. An essential tool is the following restatement of a theorem of Bollobás and Thomason: 
Lemma 36 (Lemma 5.1 from [6]). Let $K$ be a $C R G$ and define $\mathcal{H}$ to be the hereditary property of graphs, $G$, such that $G \mapsto K$. For all $p_{0} \in(0,1)$,

$$
c_{\mathcal{H}}\left(p_{0}\right)=-\log _{2}\left(p_{0}\left(1-p_{0}\right)\right) \cdot g_{K}\left(p^{*}\right)
$$

Lemma 37. Let $p_{0}$ and $\varepsilon$ be fixed such that $p_{0} \in(0,1)$ and $\varepsilon \in(0,1)$. Moreover, fix $\mathcal{B}$ to be a finite set of $C R G$ s. If $F \sim \mathbb{G}\left(n_{0}, p_{0}\right)$, the following holds a.a.s. as $n_{0} \rightarrow \infty$ : For all CRGs $K$ such that all components of $K$ lie in $\mathcal{B}$ and $F \not \neg K$, then

$$
(1+\varepsilon) g_{K}\left(p^{*}\right) \geq \frac{2 \log n_{0}}{-\log \left(p_{0}\left(1-p_{0}\right)\right) \cdot n_{0}} .
$$

Proof. For any function $\mu: \mathcal{B} \rightarrow\{0,1, \ldots\}$, define the $\mathrm{CRG}, K_{\mu}$ as follows:

$$
K_{\mu}:=\bigoplus_{B \in \mathcal{B}} \mu(B) \cdot B
$$

That is, $K_{\mu}$ consists of a disjoint union of $\mu(B)$ copies of $B$, for all $B \in$ $\mathcal{B}$. For any induced subgraph $G$ of the random graph $F \sim \mathbb{G}\left(n_{0}, p_{0}\right)$ and any CRG $K$, we will denote the event that $G$ embeds into $K$ by $[G \mapsto K]$. Additionally, let

$$
E_{\mu}:=\left[F \mapsto K_{\mu}\right]
$$

Let

$$
\mathcal{B}_{0}:=\left\{\mu: \mathcal{B} \rightarrow\{0,1, \ldots\}:(1+\varepsilon) g_{K_{\mu}}\left(p^{*}\right)<\frac{2 \log n_{0}}{-\log \left(p_{0}\left(1-p_{0}\right)\right) \cdot n_{0}}\right\} .
$$

To prove the desired claim, it is equivalent to show that the probability that $F \not \neg K_{\mu}$ for all $\mu \in \mathcal{B}_{0}$ goes to zero. That is,

$$
\lim _{n_{0} \rightarrow \infty} \mathbb{P}\left[\bigcup_{\mu \in \mathcal{B}_{0}} \overline{E_{\mu}}\right]=0 .
$$

Recall $F \sim \mathbb{G}\left(n_{0}, p_{0}\right)$. We will partition $V(F)$ by setting

$$
C:=\left\lceil 2|\mathcal{B}| \cdot \frac{1+\varepsilon / 2}{\varepsilon / 2}\right\rceil
$$


and let $I_{1}, \ldots, I_{C}$ be an equipartition of $V(F)$, i.e., $\left|I_{k}\right| \in\left\{\left\lfloor n_{0} / C\right\rfloor,\left\lceil n_{0} / C\right\rceil\right\}$ for $k \in\{1, \ldots, C\}$. Let $n_{0}^{\prime}=\left\lceil n_{0} / C\right\rceil$.

For any $B \in \mathcal{B}$, set

$$
m_{B}:=\left\lfloor\frac{(1+\varepsilon / 2) \cdot\left(n_{0} / C\right) \cdot\left(-\log \left(p_{0}\left(1-p_{0}\right)\right)\right) \cdot g_{B}\left(p^{*}\right)}{2 \log \left(n_{0} / C\right)}\right\rfloor .
$$

We expect to be able to $\left(m_{B} \cdot B\right)$-color a $\mathbb{G}\left(n_{0}^{\prime}, p_{0}\right)$ graph (hence a $\mathbb{G}\left(n_{0}^{\prime}-1, p_{0}\right)$ graph as well). Now for any $k \in\{1, \ldots, C\}$ and any $B \in \mathcal{B}$, we define the event

$$
E_{k, B}:=\left[F\left[I_{k}\right] \mapsto m_{B} \cdot B\right] .
$$

In other words, $E_{k, B}$ is the event that the subgraph of $F$ that is induced by vertices in $I_{k}$ is colorable by $m_{B}$ copies of the CRG $B$.

The induced subgraphs $F\left[I_{1}\right], \ldots, F\left[I_{C}\right]$ are each independently sampled according to the Erdős-Rényi random graph model $\mathbb{G}\left(n_{0}^{\prime}, p_{0}\right)$. Moreover, the number of events of the form $E_{k, B}$ is equal to $C \cdot|\mathcal{B}|$, which is bounded as a function of the constants $p_{0}, \varepsilon$, and $|\mathcal{B}|$.

Then by Theorem 7 and Lemma 36, since the number of vertices in each $I_{k}$ uniformly tends to $\infty$,

$$
\lim _{n_{0} \rightarrow \infty} \mathbb{P}\left[\bigcup_{k \in\{1, \ldots, C\}} \bigcup_{B \in \mathcal{B}} \overline{E_{k, B}}\right]=0 .
$$

It suffices to show that

$$
\bigcup_{\mu \in \mathcal{B}_{0}} \overline{E_{\mu}} \subseteq \bigcup_{k \in\{1, \ldots, C\}} \bigcup_{B \in \mathcal{B}} \overline{E_{k, B}}
$$

because then (14) will imply (13) and complete the proof.

Indeed, suppose $\mu \in \mathcal{B}_{0}$ and suppose $\varphi_{k, B}$ are embeddings defined by the events $E_{k, B}$, for all $k \in\{1, \ldots, C\}$ and all $B \in \mathcal{B}$. Further, for any $B \in \mathcal{B}$, let

$$
M_{B}:=\left\lfloor\frac{\mu(B)}{m_{B}}\right\rfloor .
$$


We will show that if $\sum_{B \in \mathcal{B}} M_{B} \geq C$, then $F$ can be colored by $M_{B}$ copies of $m_{B} \cdot B$, over all $B \in \mathcal{B}$, and so $F \mapsto K_{\mu}$. We begin by summing the $M_{B}$ 's.

$$
\begin{aligned}
\sum_{B \in \mathcal{B}} M_{B} & =\sum_{B \in \mathcal{B}}\left\lfloor\frac{\mu(B)}{m_{B}}\right\rfloor \\
& \geq \sum_{B \in \mathcal{B}}\left(\frac{\mu(B)}{m_{B}}-1\right) \\
& =-|\mathcal{B}|+\sum_{B \in \mathcal{B}} \frac{\mu(B) \cdot 2 \log \left(n_{0} / C\right)}{(1+\varepsilon / 2) \cdot\left(n_{0} / C\right) \cdot\left(-\log \left(p_{0}\left(1-p_{0}\right)\right)\right) \cdot g_{B}\left(p^{*}\right)} \\
& =-|\mathcal{B}|+\frac{2 C \log \left(n_{0} / C\right)}{(1+\varepsilon / 2) n_{0}} \cdot \sum_{B \in \mathcal{B}} \frac{\mu(B)}{-\log \left(p_{0}\left(1-p_{0}\right)\right) \cdot g_{B}\left(p^{*}\right)} .
\end{aligned}
$$

By Proposition 27,

$$
\sum_{B \in \mathcal{B}} M_{B}=-|\mathcal{B}|+\frac{2 C \log \left(n_{0} / C\right)}{(1+\varepsilon / 2) n_{0}} \cdot \frac{1}{-\log \left(p_{0}\left(1-p_{0}\right)\right) \cdot g_{K_{\mu}}\left(p^{*}\right)} .
$$

By (12),

$$
\begin{aligned}
\sum_{B \in \mathcal{B}} M_{B} & \geq-|\mathcal{B}|+\frac{2 C \log \left(n_{0} / C\right)}{(1+\varepsilon / 2) n_{0}} \cdot \frac{(1+\varepsilon) n_{0}}{2 \log n_{0}} \\
& =-|\mathcal{B}|+C\left(1-\frac{\log C}{\log n_{0}}\right)\left(1+\frac{\varepsilon}{2+\varepsilon}\right) \\
& =C+C\left(\frac{\varepsilon}{2+\varepsilon}-\frac{2+2 \varepsilon}{2+\varepsilon} \cdot \frac{\log C}{\log n_{0}}\right)-|\mathcal{B}|
\end{aligned}
$$

With $\varepsilon$ fixed and $n_{0} \gg C \gg|\mathcal{B}|$, we have $\sum_{B \in \mathcal{B}} M_{B} \geq C$, as desired. Thus, the index set $\{1, \ldots, C\}$ may be partitioned as

$$
\{1, \ldots, C\}=\bigcup_{B \in \mathcal{B}} S_{B}
$$

so that $\left|S_{B}\right| \leq M_{B}$, for each $B \in \mathcal{B}$. Also, for each $B \in \mathcal{B}$, we combine the embeddings $\varphi_{B, k}$ for all $k \in S_{B}$ to form an embedding

$$
F\left[\bigcup_{k \in S_{B}} I_{k}\right] \mapsto\left(\left|S_{B}\right| \cdot m_{B}\right) \cdot B \subseteq \mu(B) \cdot B .
$$


Combining all such embeddings, $F \mapsto K_{\mu}$, as desired. So

$\bigcap_{k \in\{1, \ldots, C\}} \bigcap_{B \in \mathcal{B}} E_{k, B} \subseteq \bigcap_{\mu \in \mathcal{B}_{0}} E_{\mu} \quad \Longleftrightarrow \quad \bigcup_{\mu \in \mathcal{B}_{0}} \overline{E_{\mu}} \subseteq \bigcup_{k \in\{1, \ldots, C\}} \bigcup_{B \in \mathcal{B}} \overline{E_{k, B}}$.

This completes the proof of the desired claim.

Finally, we are ready to prove Theorem 4.

3.3. Proof of Theorem 4. Formally, our goal is to show that for each $\varepsilon \in(0,1)$, the following occurs a.a.s. as $n_{0} \rightarrow \infty$ :

$$
\sup _{p \in I}\left|\operatorname{ed}_{\mathcal{H}}(p)\left(\frac{2 \log n_{0}}{n_{0}} \cdot \min \left\{\frac{p}{-\log \left(1-p_{0}\right)}, \frac{1-p}{-\log p_{0}}\right\}\right)^{-1}-1\right|<\varepsilon,
$$

where

$$
I=\left\{\begin{aligned}
{[0,1], } & \text { if } p^{*} \in\left[1-\varphi^{-1}, \varphi^{-1}\right] \\
{[1 / 3,1], } & \text { if } p^{*} \in\left[0,1-\varphi^{-1}\right) \\
{[0,2 / 3], } & \text { if } p^{*} \in\left(\varphi^{-1}, 1\right]
\end{aligned}\right.
$$

We first establish an upper bound for $\operatorname{ed}_{\mathcal{H}}(p)$ for all $p \in[0,1]$. By the main result in [5], if $F \sim \mathbb{G}\left(n_{0}, p_{0}\right)$, then a.a.s. as $n_{0} \rightarrow \infty$,

$$
\begin{gathered}
\chi(F)-1 \geq(1-\varepsilon / 2) \frac{n_{0}}{2 \log _{1 /\left(1-p_{0}\right)} n_{0}}, \\
\chi\left(F^{c}\right)-1 \geq(1-\varepsilon / 2) \frac{n_{0}}{2 \log _{1 / p_{0}} n_{0}} .
\end{gathered}
$$

Clearly $F$ does not map into $\chi(F)-1$ white vertices or $\chi\left(F^{c}\right)-1$ black vertices. By, for example Theorem 10 , and the fact that $1 /(1-\varepsilon / 2)<$ $1+\varepsilon$, then the following occurs a.a.s.:

$$
\begin{aligned}
\operatorname{ed}_{\mathcal{H}}(p) & \leq(1+\varepsilon) \min \left\{\frac{p}{n_{0} /\left(2 \log _{1 /\left(1-p_{0}\right)} n_{0}\right)}, \frac{1-p}{n_{0} /\left(2 \log _{1 / p_{0}} n_{0}\right)}\right\} \\
& =(1+\varepsilon) \frac{2 \log n_{0}}{n_{0}} \cdot \min \left\{\frac{p}{-\log \left(1-p_{0}\right)}, \frac{1-p}{-\log p_{0}}\right\} .
\end{aligned}
$$

We now find a lower bound to match Inequality (16) over the interval $I$ as stated above. We will do this by finding the lower bound for $p \in(1 / 3,2 / 3)$ and then use concavity show how this extends to $I$ in 
the various cases.

We will choose a $\tilde{p} \in(1 / 3,2 / 3)$ depending on the case:

$$
\tilde{p}:=\left\{\begin{aligned}
p^{*}, & \text { if } p^{*} \in(1 / 3,2 / 3) \\
1 / 3+\varepsilon / 9, & \text { if } p^{*} \in(0,1 / 3] \\
2 / 3-\varepsilon / 9, & \text { if } p^{*} \in[2 / 3,1)
\end{aligned}\right.
$$

Let $K=K(\tilde{p}) \in \mathcal{K}_{\mathcal{H}}$ be a $\tilde{p}$-core CRG that $\operatorname{satisfies} \operatorname{ed}_{\mathcal{H}}(\tilde{p})=g_{K}(\tilde{p})$, as guaranteed by Theorem $[10$.

Since $\tilde{p} \in(1 / 3,2 / 3)$, Lemma 31 gives that there exists a sub-CRG $K^{\prime}=K^{\prime}(\tilde{p}, \varepsilon / 4)$ of $K$ so that

$$
g_{K}(\tilde{p}) \leq g_{K^{\prime}}(\tilde{p}) \leq(1+\varepsilon / 4) g_{K}(\tilde{p})
$$

and whose components lie in some finite set $\mathcal{B}=\mathcal{B}(\tilde{p}, \varepsilon / 4)$ of CRGs.

The function $g_{K^{\prime}}:[0,1] \rightarrow[0,1]$ is concave-down. To see this, let $M_{K^{\prime}}(p)$ be the matrix defined by the CRG $K$. Let $p_{1}, p_{2} \in[0,1]$, $t \in[0,1]$, and let $\mathbf{x} \in \Delta_{K^{\prime}}$ be the vector that witnesses the value of $g_{K^{\prime}}$ at $t p_{1}+(1-t) p_{2}$

$$
\begin{aligned}
g_{K^{\prime}}\left(t p_{1}+(1-t) p_{2}\right) & =\left\langle\mathbf{x}, M_{K^{\prime}}\left(t p_{1}+(1-t) p_{2}\right) \mathbf{x}\right\rangle \\
& =t \cdot\left\langle\mathbf{x}, M_{K^{\prime}}\left(p_{1}\right) \mathbf{x}\right\rangle+(1-t) \cdot\left\langle\mathbf{x}, M_{K^{\prime}}\left(p_{2}\right) \mathbf{x}\right\rangle \\
& \geq t \cdot g_{K^{\prime}}\left(p_{1}\right)+(1-t) \cdot g_{K^{\prime}}\left(p_{2}\right),
\end{aligned}
$$

establishing the concavity of $g_{K^{\prime}}$. Since $g_{K^{\prime}}(0), g_{K^{\prime}}(1) \geq 0$, the graph of $g_{K^{\prime}}$ lies above the line segment from $(0,0)$ to $\left(\tilde{p}, g_{K^{\prime}}(\tilde{p})\right)$ to $(1,0)$. So

$$
g_{K^{\prime}}(p) \geq g_{K^{\prime}}\left(p^{*}\right) \cdot \min \left\{\frac{p}{p^{*}}, \frac{1-p}{1-p^{*}}\right\}
$$

Also by Lemma 37 (recall the definition of $p^{*}$ from (91)), the following is true a.a.s.:

$$
g_{K^{\prime}}\left(p^{*}\right) \geq(1-\varepsilon / 4) \cdot \frac{2 \log n_{0}}{-\log \left(p_{0}\left(1-p_{0}\right)\right) \cdot n_{0}} .
$$


Combining (17), (18), and (19),

$$
\begin{aligned}
\operatorname{ed}_{\mathcal{H}}(\tilde{p}) & =g_{K}(\tilde{p}) \\
& \geq \frac{1}{1+\varepsilon / 4} g_{K^{\prime}}(\tilde{p}) \\
& \geq \frac{1}{1+\varepsilon / 4} g_{K^{\prime}}\left(p^{*}\right) \cdot \min \left\{\frac{\tilde{p}}{p^{*}}, \frac{1-\tilde{p}}{1-p^{*}}\right\} \\
& \geq \frac{1-\varepsilon / 4}{1+\varepsilon / 4} \cdot \frac{2 \log n_{0}}{-\log \left(p_{0}\left(1-p_{0}\right)\right) \cdot n_{0}} \cdot \min \left\{\frac{\tilde{p}}{p^{*}}, \frac{1-\tilde{p}}{1-p^{*}}\right\} \\
& \geq(1-\varepsilon / 2) \frac{2 \log n_{0}}{-\log \left(p_{0}\left(1-p_{0}\right)\right) \cdot n_{0}} \cdot \min \left\{\frac{\tilde{p}}{p^{*}}, \frac{1-\tilde{p}}{1-p^{*}}\right\}
\end{aligned}
$$

Case 1. $p_{0} \in\left(1-\varphi^{-1}, \varphi^{-1}\right) \Longleftrightarrow p^{*} \in(1 / 3,2 / 3)$.

In this case, $\tilde{p}=p^{*}$. By (20) and the concavity of $\operatorname{ed}_{\mathcal{H}}(p)$,

$$
\begin{aligned}
\operatorname{ed}_{\mathcal{H}}\left(p^{*}\right) & \geq(1-\varepsilon / 2) \frac{2 \log n_{0}}{-\log \left(p_{0}\left(1-p_{0}\right)\right) \cdot n_{0}} \\
\operatorname{ed}_{\mathcal{H}}(p) & \geq(1-\varepsilon / 2) \frac{2 \log n_{0}}{-\log \left(p_{0}\left(1-p_{0}\right)\right) \cdot n_{0}} \cdot \min \left\{\frac{p}{p^{*}}, \frac{1-p}{1-p^{*}}\right\} \\
& =(1-\varepsilon / 2) \frac{2 \log n_{0}}{n_{0}} \cdot \min \left\{\frac{p}{-\log \left(1-p_{0}\right)}, \frac{1-p}{-\log p_{0}}\right\} .
\end{aligned}
$$

This satisfies (15) for all $p \in[0,1]$, completing the proof in this case.

Case 2. $p_{0} \in\left(0,1-\varphi^{-1}\right] \Longleftrightarrow p^{*} \in(0,1 / 3]$.

In this case, $\tilde{p}=1 / 3+\varepsilon / 9>p^{*}$. By (20) and the concavity of ed $\operatorname{di}_{\mathcal{H}}$,

$$
\begin{aligned}
& \operatorname{ed}_{\mathcal{H}}(\tilde{p}) \geq(1-\varepsilon / 2) \frac{2 \log n_{0}}{-\log \left(p_{0}\left(1-p_{0}\right)\right) \cdot n_{0}} \cdot \frac{1-\tilde{p}}{1-p^{*}} \\
& \operatorname{ed}_{\mathcal{H}}(p) \geq(1-\varepsilon / 2) \frac{2 \log n_{0}}{-\log \left(p_{0}\left(1-p_{0}\right)\right) \cdot n_{0}} \cdot \frac{1-\tilde{p}}{1-p^{*}} \cdot \min \left\{\frac{p}{\tilde{p}}, \frac{1-p}{1-\tilde{p}}\right\} .
\end{aligned}
$$

Substituting $\tilde{p}=1 / 3+\varepsilon / 9$ and $p=1 / 3$,

$$
\begin{aligned}
\operatorname{ed}_{\mathcal{H}}(1 / 3) & \geq(1-\varepsilon / 2) \frac{2 \log n_{0}}{-\log \left(p_{0}\left(1-p_{0}\right)\right) \cdot n_{0}} \cdot \frac{2 / 3-\varepsilon / 9}{1-p^{*}} \cdot \frac{1 / 3}{1 / 3+\varepsilon / 9} \\
& =\frac{(1-\varepsilon / 2)(1 / 3-\varepsilon / 18)}{1 / 3+\varepsilon / 9} \cdot \frac{2 \log n_{0}}{n_{0}} \cdot \frac{2 / 3}{-\log p_{0}} \\
& \geq(1-\varepsilon) \frac{2 \log n_{0}}{n_{0}} \cdot \frac{2 / 3}{-\log p_{0}} .
\end{aligned}
$$


Again by concavity,

$$
\operatorname{ed}_{\mathcal{H}}(p) \geq(1-\varepsilon) \frac{2 \log n_{0}}{n_{0}} \cdot \frac{2 / 3}{-\log p_{0}} \cdot \min \left\{\frac{p}{1 / 3}, \frac{1-p}{2 / 3}\right\} .
$$

This matches the upper bound (16) for all $p \in[1 / 3,1]$ and, in fact, if $p^{*}=1 / 3$, then it matches the upper bound for all $p \in[0,1]$. This completes the proof in this case.

Case 3. $p_{0} \in\left[\varphi^{-1}, 1\right) \Longleftrightarrow p^{*} \in[2 / 3,1)$.

This case may be shown with a similar argument as Case 2 . In this case, $\tilde{p}=2 / 3-\varepsilon / 9<p^{*}$. By (20) and the concavity of $\operatorname{ed}_{\mathcal{H}}$,

$$
\operatorname{ed}_{\mathcal{H}}(p) \geq(1-\varepsilon) \frac{2 \log n_{0}}{n_{0}} \cdot \frac{1 / 3}{-\log p_{0}} \cdot \min \left\{\frac{p}{2 / 3}, \frac{1-p}{1 / 3}\right\} .
$$

This matches the upper bound (16) for all $p \in[0,2 / 3]$ and if $p^{*}=2 / 3$, then it matches the upper bound for all $p \in[0,1]$. This completes the proof in this case and the proof of Theorem 4 .

\section{Discussion}

In the process of proving the main result, Theorem 4, we have developed a number of observations that apply generally to computing edit distance functions. Lemma 20 gives a general condition for which a CRG is $p$-prohbited and Lemma 23 shows that for $p \in\left[1-\varphi^{-1}, \varphi^{-1}\right]$, the only CRGs that need to be considered are dalmatian sets and their complements.

In this section, we discuss some other general results.

\subsection{Defining the edit distance function with a finite set of} CRGs. The following was conjectured by the first author:

Conjecture 38 ([11]). Let $\mathcal{H}$ be a nontrivial hereditary property. For every $\varepsilon>0$ there exists a finite set of $C R G s \mathcal{K}^{\prime}=\mathcal{K}^{\prime}(\varepsilon, \mathcal{H})$ such that

$$
\operatorname{ed}_{\mathcal{H}}(p)=\min \left\{g_{K}(p): K \in \mathcal{K}^{\prime}\right\}, \quad \text { for all } p \in(\varepsilon, 1-\varepsilon) .
$$

Note that this is stronger than the Marchant-Thomason result in Theorem [10] which says that, for every $p$ there is a finite set of CRGs that define $\operatorname{ed}_{\mathcal{H}}(p)$. Conjecture 38 asserts that a single finite set will define $\operatorname{ed}_{\mathcal{H}}(p)$ for all $p$ an arbitrary open interval in $(0,1)$. In Theorem 39, we provide a partial answer by showing that the conjecture is 
true for $\varepsilon \geq 1-\varphi^{-1}$.

Theorem 39. Let $\mathcal{H}$ be a nontrivial hereditary property. There exists a finite set of $C R G s, \mathcal{K}^{\prime}=\mathcal{K}^{\prime}(\mathcal{H})$, such that

$$
\operatorname{ed}_{\mathcal{H}}(p)=\min \left\{g_{K}(p): K \in \mathcal{K}^{\prime}\right\}, \quad \text { for all } p \in\left[1-\varphi^{-1}, \varphi^{-1}\right] .
$$

Proof. By Lemma 23, the only $p$-core CRGs are denoted $\mathcal{D}_{p}$ and consist of components which are dalmatian CRGs if $p \in\left[1-\varphi^{-1}, 1 / 2\right)$, complements of dalmatian CRGs if $p \in\left(1 / 2, \varphi^{-1}\right]$, and CRGs with only gray edges if $p=1 / 2$.

It is easy to see that if $p=1 / 2$ and $\mathcal{H}=\operatorname{Forb}(\mathcal{F})$, then for any $F \in \mathcal{F}$ such that $F \not \rightarrow K$, the number of vertices of $K$ is bounded by $\chi(F)+\chi\left(F^{c}\right)$, so the number of such CRGs is finite. We will now show that a finite set of CRGs suffice for $p \in\left[1-\varphi^{-1}, 1 / 2\right)$. The case $p \in\left(1 / 2, \varphi^{-1}\right]$ follows by symmetry. Note that $\mathcal{D}_{p}$ is the same for all $p \in[0,1 / 2)$ (that is, CRGs whose components are all dalmatian CRGs) so we denote $\mathcal{D}_{0, \mathcal{H}}:=\mathcal{D}_{p} \cap \mathcal{K}_{\mathcal{H}}$.

Write $\mathcal{H}=\operatorname{Forb}(\mathcal{F})$ and, for a contradiction, let $\left\{p_{k}\right\}_{k=1}^{\infty} \subset[1-$ $\left.\varphi^{-1}, 1 / 2\right)$ be an infinite set and let $\mathcal{K}^{\prime}:=\left\{K_{k}\right\}_{k=1}^{\infty} \subset \mathcal{D}_{0, \mathcal{H}}$ an infinite set of CRGs such that $K_{k}$ is $p_{k}$-core and $g_{K_{k}}\left(p_{k}\right)=\operatorname{ed}_{\mathcal{H}}\left(p_{k}\right)$.

For all $k \geq 1$, with $D_{i}$ denoting a dalmatian CRG of order $i$, we may write

$$
K_{k}=D_{c_{k}^{(1)}} \oplus \cdots \oplus D_{c_{k}^{\left(\ell_{k}\right)}} \oplus\left(w_{k} \cdot D_{\infty}\right)
$$

where $w_{k}, \ell_{k}, c_{k}^{(1)}, \ldots, c_{k}^{\left(\ell_{k}\right)}$ are nonnegative integers and $c_{k}^{(1)} \geq \cdots \geq$ $c_{k}^{\left(\ell_{k}\right)}$.

For all $k \geq 1, w_{k}+\ell_{k} \leq|V(F)|$ for any $F \in \mathcal{F}$ because otherwise $F \mapsto K_{k}$ by embedding each vertex of $F$ into a different component of $K_{k}$. Thus, $\ell_{k}$ is bounded by an absolute constant $\ell=\ell(\mathcal{H})$. So we associate each $K_{k}$ in (21) with the $(\ell+1)$-tuple

$$
\left(c_{k}^{(1)}, \ldots, c_{k}^{(\ell)} ; w_{k}\right)
$$

where $c_{k}^{\left(\ell_{k}+1\right)}=\cdots=c_{k}^{(\ell)}=0$ if $\ell>\ell_{k}$.

Because $\mathcal{K}^{\prime}$ is infinite, there exists a maximum $m \in\{1, \ldots, \ell\}$ such that $\sup _{k}\left\{c_{k}^{(1)}\right\}=\cdots=\sup _{k}\left\{c_{k}^{(m)}\right\}=\infty$. That is, if $m<\ell$, then 
$\sup _{k}\left\{c_{k}^{(m+1)}\right\}<\infty$. Thus, there is a fixed (possibly empty) tuple $\left(c_{*}^{(m+1)}, \ldots, c_{*}^{(\ell)} ; w_{*}\right)$ and an infinite subsequence $k_{1}, k_{2}, \ldots$ such that $K_{k_{i}}$ is associated with $(\ell+1)$-tuple $\left(c_{k_{i}}^{(1)}, \ldots, c_{k_{i}}^{(m)}, c_{*}^{(m+1)}, \ldots, c_{*}^{(\ell)} ; w_{*}\right)$.

With this choice of $\left(c_{*}^{(m+1)}, \ldots, c_{*}^{(\ell)} ; w_{*}\right)$, if $\ell^{\prime}$ is the largest entry such that $c_{*}^{\left(\ell^{\prime}\right)} \geq 1$, then define

$$
K_{*}=D_{c_{k}^{(m+1)}} \oplus \cdots \oplus D_{c_{k}^{\left(\ell^{\prime}\right)}} \oplus\left(\left(m+w_{*}\right) \cdot D_{\infty}\right) .
$$

We claim that $K_{*} \in \mathcal{D}_{0, \mathcal{H}} \subseteq \mathcal{K}_{\mathcal{H}}$.

If not, then there exists some $F \in \mathcal{F}$ and some embedding $\phi$ : $V(F) \rightarrow V\left(K_{*}\right)$. Let $A_{1}, \ldots, A_{m}$ be the preimages of the first $m$ copies of $D_{\infty}$ under $\varphi$, respectively. Then $A_{1}, \ldots, A_{m}$ are independent sets in $F$.

Let $k$ be sufficiently large so that $c_{1}^{k}, \ldots, c_{m}^{k} \geq|V(F)|$. Then we define $\phi^{\prime}: V(F) \rightarrow V\left(K_{k}\right)$ by instead sending the vertices of $A_{i}$ to distinct vertices of the dalmatian set $D_{c_{i}^{k}}$, for each $i \in\{1, \ldots, m\}$. As a result, $\phi^{\prime}$ is an embedding of $F$ into $K_{k}$, a contradiction.

Finally by Proposition 27 and Remark 22 , for all $p \in(0,1 / 2)$ and $k$ chosen as above,

$$
\begin{aligned}
g_{K_{k}}(p)^{-1} & =\frac{w}{p}+\sum_{i=1}^{m} \frac{1}{p+(1-2 p) / c_{i}^{k}}+\sum_{i=1}^{\ell-m} \frac{1}{p+(1-2 p) / c_{i}} \\
& <\frac{w+m}{p}+\sum_{i=1}^{\ell-m} \frac{1}{p+(1-2 p) / c_{i}} \\
& =g_{K_{*}}(p)^{-1}
\end{aligned}
$$

The fact that $g_{K_{*}}\left(p_{k}\right)<g_{K_{k}}\left(p_{k}\right)=\operatorname{ed}_{\mathcal{H}}\left(p_{k}\right)$ contradicts $K_{*} \in \mathcal{K}_{\mathcal{H}}$, hence the original assumption that $\mathcal{K}^{\prime}$ is infinite.

4.2. Paths. In Proposition 35, it is established that $P_{d}$ is $p$-prohibited for $p \in\left[\frac{1}{1+2 \cos (\pi /(d+1))}, 1-\frac{1}{1+2 \cos (\pi /(d+1))}\right]$. In the case of $d=3, P_{3}$ is $p$-prohibited for $p$ in the interval

$$
[\sqrt{2}-1,2-\sqrt{2}] \approx[0.414214,0.585786] .
$$


However, Lemma 23 establishes that $P_{3}$ is $p$-prohibited if and only if $p$ is in the interval

$$
\left[1-\varphi^{-1}, \varphi^{-1}\right]=\left[\frac{3-\sqrt{5}}{2}, \frac{\sqrt{5}-1}{2}\right] \approx[0.381966,0.618034] .
$$

We ask whether $P_{d}$ is $p$-prohibited over a larger interval than given in (11). See Table 1 for small values.

\begin{tabular}{|c|c|c|}
\hline$d$ & $(1+2 \cos (\pi /(d+1)))^{-1}$ & $1-(1+2 \cos (\pi /(d+1)))^{-1}$ \\
\hline 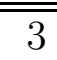 & $=\sqrt{2}-1 \approx 0.414214$ & $2-\sqrt{2} \approx 0.585786$ \\
\hline 4 & $(3-\sqrt{5}) / 2 \approx 0.381966$ & $(\sqrt{5}-1) / 2 \approx 0.618034$ \\
\hline 5 & $(\sqrt{3}-1) / 2 \approx 0.366025$ & $(3-\sqrt{3}) / 2$ \\
\hline 6 & $\approx 0.356896$ & $\approx 0.643104$ \\
\hline 7 & $\approx 0.351153$ & $\approx 0.648847$ \\
\hline 8 & $\approx 0.347296$ & $\approx 0.652704$ \\
\hline 9 & $\approx 0.344577$ & $\approx 0.655423$ \\
\hline 10 & $\approx 0.342585$ & $\approx 0.657415$ \\
\hline 11 & $\approx 0.341081$ & $\approx 0.658919$ \\
\hline 12 & $\approx 0.339918$ & $\approx 0.660082$ \\
\hline 13 & $\approx 0.339000$ & $\approx 0.661000$ \\
\hline 14 & $\approx 0.338261$ & $\approx 0.661739$ \\
\hline 15 & $\approx 0.337659$ & $\approx 0.662341$ \\
\hline
\end{tabular}

TABLE 1. Table for endpoints of an interval where $P_{d}$ is prohibited.

Question 40. For $d \geq 4$, what is the largest interval over which $P_{d}$ is p-prohibited?

\section{QUeSTIONS AND FUTURE WORK}

5.1. $p$-core CRGs. Lemma 23 classifies all $p$-core CRGs on the interval $\left[1-\varphi^{-1}, \varphi^{-1}\right]$.

Question 41. For which $a \in\left(0,1-\varphi^{-1}\right)$ does there exist an elementary classification of all $p$-core CRGs for all $p \in[a, 1-a]$ ? Additionally, are all sufficiently large connected p-core CRGs either dalmatian CRGs (if $p \leq 1 / 2$ ) or the complement of a dalmatian $C R G$ (if $p \geq 1 / 2$ )? 
A crucial part of the proof of Theorem 4 is Lemma 31, which establishes that, for $p \in(1 / 3,2 / 3)$, a $p$-core $C R G$ can be approximated so that the $g$ function does not increase by much, but the components are bounded.

Question 42. Does Lemma 31 hold if the interval $(1 / 3,2 / 3)$ is widened to $(a, 1-a)$ for some $a \in(0,1 / 3)$ ?

5.2. Inhomogeneous random graphs. Since the development of graph limits, inhomogeneous generalizations $\mathbb{G}(n, W)$ of the Erdős-Rényi random graph models have emerged as a topic of research interest (see [9]). Here, $W: \Omega^{2} \rightarrow[0,1]$ is a graphon, which is a symmetric measurable function where $\Omega$ is a probability space, frequently $[0,1]$ equipped with the Lebesgue measure. To form a $W$-random graph $G \sim \mathbb{G}(n, W)$, sample $n$ elements $x_{1}, \ldots, x_{n} \sim \Omega$ independently and form a graph on $\{1, \ldots, n\}$ by adding edge $i j$ independently with probability $W\left(x_{i}, x_{j}\right)$. We may also generate a sequence of $W$-random graphs $\left(G_{n}\right)_{n=1}^{\infty} \sim$ $\mathbb{G}(\mathbb{N}, W)$ adding the vertices corresponding to $x_{1}, x_{2}, \ldots$, one at a time.

There are several questions we may ask related to the edit distance problem and inhomogeneous random graphs.

First, note that Theorem 4 implies that with $p_{0} \in\left[1-\varphi^{-1}, \varphi^{-1}\right]$ and $\left(F_{n}\right) \sim \mathbb{G}\left(\mathbb{N}, p_{0}\right)$, then a.a.s.,

$$
\lim _{n \rightarrow \infty} \sup _{p \in[0,1]} \frac{\operatorname{ed}_{\operatorname{Forb}\left(F_{n+1}\right)}(p)}{\operatorname{ed}_{\operatorname{Forb}\left(F_{n}\right)}(p)}=1 .
$$

Question 43. For what graphons $W$ does Equation (22) a.a.s. hold for $\left(F_{n}\right) \sim \mathbb{G}(\mathbb{N}, W)$ ?

For the next question, we note the following expression for the distance from a homogeneous random graph to a hereditary property. Combining Theorems 2 and 10, we see that if $\mathcal{H}$ is a (fixed) nontrivial hereditary property and $p \in[0,1]$, then with $G_{n} \sim \mathbb{G}(n, p)$,

$$
\lim _{n \rightarrow \infty} \mathbb{E}\left[\operatorname{dist}\left(G_{n}, \mathcal{H}\right)\right]=\inf _{K \in \mathcal{K}_{\mathcal{H}}} g_{K}(p)=\min _{K \in \mathcal{K}_{\mathcal{H}}} g_{K}(p) .
$$


Question 44. If instead $G_{n} \sim \mathbb{G}(n, W)$, is there a similar expression for $\lim \sup _{n \rightarrow \infty} \mathbb{E}\left[\operatorname{dist}\left(G_{n}, \mathcal{H}\right)\right]$ ? In particular, can we extend the functions $g_{K}(\cdot)$ from $[0,1]$ to the set of all graphons so that Equation (23) holds?

5.3. Acknowledgements. The authors would like to thank Jan Hladký and Josh Cooper for helpful comments.

\section{REFERENCES}

[1] V. E. Alekseev. Hereditary classes and coding of graphs. Problemy Kibernet., (39):151-164, 1982.

[2] Noga Alon and Uri Stav. The maximum edit distance from hereditary graph properties. J. Combin. Theory Ser. B, 98(4):672-697, 2008.

[3] Noga Alon and Uri Stav. What is the furthest graph from a hereditary property? Random Structures Algorithms, 33(1):87-104, 2008.

[4] József Balogh and Ryan Martin. Edit distance and its computation. Electron. J. Combin., 15(1):Research Paper 20, 27, 2008.

[5] B. Bollobás. The chromatic number of random graphs. Combinatorica, 8(1):4955, 1988.

[6] Béla Bollobás and Andrew Thomason. The structure of hereditary properties and colourings of random graphs. Combinatorica, 20(2):173-202, 2000.

[7] Andries E. Brouwer and Willem H. Haemers. Spectra of graphs. Universitext. Springer, New York, 2012.

[8] Dragoš Cvetković, Peter Rowlinson, and Slobodan Simić. An introduction to the theory of graph spectra, volume 75 of London Mathematical Society Student Texts. Cambridge University Press, Cambridge, 2010.

[9] László Lovász. Large networks and graph limits, volume 60 of American Mathematical Society Colloquium Publications. American Mathematical Society, Providence, RI, 2012.

[10] Edward Marchant and Andrew Thomason. Extremal graphs and multigraphs with two weighted colours. In Fete of combinatorics and computer science, volume 20 of Bolyai Soc. Math. Stud., pages 239-286. János Bolyai Math. Soc., Budapest, 2010.

[11] Ryan R. Martin. The edit distance in graphs: methods, results, and generalizations. In Recent trends in combinatorics, volume 159 of IMA Vol. Math. Appl., pages 31-62. Springer, [Cham], 2016.

[12] Alexander Sidorenko. Boundedness of optimal matrices in extremal multigraph and digraph problems. Combinatorica, 13(1):109-120, 1993.

[13] Andrew Thomason. Graphs, colours, weights and hereditary properties. In Surveys in combinatorics 2011, volume 392 of London Math. Soc. Lecture Note Ser., pages 333-364. Cambridge Univ. Press, Cambridge, 2011.

Department of Mathematics, Iowa State University, Ames, IA 500112064

E-mail address: rymartin@iastate.edu, awnr@iastate.edu 